\title{
Exploring the Characteristics of High-Speed Rail and Air Transportation Networks in China: A Weighted Network Approach
}

Author(s): Qingyu Qi, Oh Kyoung Kwon

Source: Journal of International Logistics and Trade 2021; 19(2):96-114

Published by: Jungseok Research Institute of International Logistics and Trade, Inha University

DOI: https://doi.org/10.24006/jilt.2021.19.2.096

Journal of International Logistics and Trade is an official journal published by Jungseok Research Institute of International Logistics and Trade, Inha University, Korea. JILT welcomes manuscripts that advance the practice and science of logistics, trade, and other related fields.

Frequency: Quarterly (March, June, September, December)

Stable URL: https://www.ejilt.org

Jungseok Research Institute of International Logistics and Trade is a specialized academic research institute representing Inha University and Inha Foundation in Korea. The institute aims to become a representative institute in Northeast Asia in the research of logistics and trade.

Stable URL: https://jrieng.inha.ac.kr

(C) Copyright. Jungseok Research Institute of International Logistics and Trade.

This is an Open-Access article distributed under the terms of the Creative Commons Attribution NonCommercial License (http://creativecommons.org/licenses/by-nc/4.0/) which permits unrestricted noncommercial use, distribution, and reproduction in any medium, provided the original work is properly cited 



\title{
ARTICLE
}

\section{Exploring the Characteristics of High-Speed Rail and Air Transportation Networks in China: A Weighted Network Approach}

\author{
Qingyu Qi, Oh Kyoung Kwon \\ Graduate School of Logistics, Inha University, Incheon 22212, Korea
}

Received June 08, 2021
Revised June 24, 2021
Accepted June 30, 2021

*Corresponding author: Oh Kyoung Kwon
Graduate School of Logistics, Inha
University, Incheon 22212, Korea
Tel: +82-32-860-7765
E-mail: scm@inha.ac.kr

\section{Introduction}

China's high-speed railway transportation industry has gradually developed ever since the Beijing-Tianjin inter-city train began operating in 2008. According to the 2019 Statistical Bulletin of the China Railway Corporation (MOT 2020), the highspeed railway passenger' volume has maintained its robust growth. In 2019, passenger traffic was 2.66 billion, having seen year-over-year rises of $8.4 \%$. National railway passenger turnover reached 1.470664 trillion person-kilometers, with a $4 \%$ increase compared to the previous year.

Higher speed is the most significant and essential feature of high-speed rail (HSR) that differentiates it from other railways. HSR not only improves the quality of the traditional railway service but also affects regional economic and social activities (Chen 2012; Li and Rong 2020). The first 'bullet trains' began operating in Japan in the 1960s; the average yearly passenger traffic of the 'Shinkansen' reached nearly 200 million passengers in the first three decades of operation (MLIT 2012). The success of the 'Shinkansen' impacted European HSR construction, and European countries have also built HSR and put it into operation in the 1990s.

Over 29,000 km of dedicated HSR lines have been put into operation in China since 2008, which is far more than the total length of high-speed railway lines in the rest of the world. Moreover, the "eight vertical and eight horizontal" network structure of China's HSR network covers most of the large and medium-sized cities (Lawrence 2019).
Keywords Weighted complex network, Weighted centrality, Network robustness, China

high-speed rail network, Air transportation network

\begin{abstract}
This study explores the characteristics of high-speed rail (HSR) and air transrelated studies have largely implemented unweighted (binary) network analysis, or have constructed a weighted network, limited by unweighted centrality measures. This study applies weighted centrality measures (mean association [MA], triangle betweenness centrality [TBC], and weighted harmonic centrality [WHC]) to represent traffic dynamics in represent passenger traffic. The spatial distribution of centrality results is visualized by using ArcGIS 10.2. Moreover, we analyze the network robustness of HSR, air transportation, and multimodal networks by measuring weighted efficiency (WE) subjected to the highest a higher MA are concentrated in the Yangtze River Delta and the Pearl River Delta; cities with a higher TBC are mostly provincial capitals or regional centers; and cities with a higher centrality results is found between HSR and air transportation networks. There is a little bit of difference in eastern cities; cities in the central region have complementary roles in HSR robustness analysis results show that the multimodal network, which includes both airports and high-speed rail stations, has the best connectivity and shows robustness.
\end{abstract}


Many studies (Behrens and Pels 2012; Wang et al. 2018) found that HSR inevitably influences the air transportation market in terms of distance, travel time, fares, and services. There is also a clear overlap between HSR and air transportation networks in China from a construction layout perspective. But these two modes of transportation are indispensable parts of China's comprehensive transportation system, and play an important role in forming the urban linkage system.

According to preliminary data on International Civil Aviation Organization (ICAO) scheduled flights, 4.1 billion passengers used air transportation to meet their business and tourism needs in 2017 (ICAO 2018). In the last few years, the scale of China's aviation network has increased rapidly. According to the Statistical Bulletin of Civil Aviation Industry Development of China, the passenger throughput of civil aviation airports reached 1.265 billion passengers in 2018, increasing $10.2 \%$ over 2017. In addition, Beijing, Shanghai, and Guangzhou airports accounted for $23.3 \%$ of all domestic airport' passengers.

This study mainly uses the weighted complex network approach to explore the characteristics of China's HSR networks, where nodes represent cities, and links represent passenger traffic. We compare centrality results from cities with HSR and air transportation networks to investigate the relationship between the two. Furthermore, using weighted efficiency (WE), we measure network robustness of HSR, air transportation, and multimodal networks to reflect the networks' abilities to avoid malfunctions under emergency events. This study offers practical implications to improve transportation network efficiency and scientific decision-making for infrastructure investment.

\section{Literature review}

In the competitive global market, no individual, organization, or company acts in isolation. Social network analysis (SNA) has been proposed to study the patterns and regularity of relationships among individuals interacting in a social environment (Scott 1988). Based on graph theory, it can be generalized as a network made up of "nodes" (vertices) and "links" (edges) connecting different pairs of nodes (Diestel 1997).

During the past few years, SNA has been used as a method to explore the characteristics of various transportation networks (Barrat et al. 2004; Du et al. 2017).

\subsection{Social network analysis of air transportation networks}

Some of the previous studies explored the worldwide airport network topological structure (Guimerà et al. 2005; Lordan et al. 2014). Guimerà et al. (2005) found that the worldwide air transportation structure is a small-world network that shows a scale-free feature in its distribution. In addition, nodes with more connections are not always the most "central" ones in the network.

Some studies explored air transportation networks on a countrywide scale, such as the ones in the United States (Xu and Harriss 2008), China (Jiang et al. 2017), and India (Bagler 2008). Xu and Harriss (2008) investigated a US air transportation network where nodes represent cities, and links represent airline connections weighted by average daily traffic. They found a rich-club phenomenon such that to a large degree, cities tend to form interconnections to sustain high traffic volumes. Bagler (2008) explored the properties and topological structure of an airport network in India by using metrics of SNA, such as degree, average shortest path length, and clustering coefficient.

A few studies considered the weight of the links between nodes to analyze air transportation networks, usually considering the number of flights or amount of passenger flow as the weight (Chung et al. 2020; Du et al. 2017). Du et al. (2017) studied Chinese air transportation as a weighted network, treating all airports located in provinces as nodes, with links denoting the flights, and they found the network exhibits a small-world property, with a homogeneous structure, and disassortative mixing. Chung et al. (2020) explored the Asian international passenger aviation market's network characteristics in 2014 and 2018 by using weighted network analysis. They mainly implemented some new weighted centrality measures (mean association [MA], triangle betweenness centrality [TBC], weighted pagerank [WPR], and reverse weighted pagerank [RWPR]) to overcome traditional SNA limitations.

\subsection{Social network analysis of high-speed rail networks}

Convenience, fast speed, and high-quality services are the main features of HSR that make HSR a new option for travelers. Some studies employed centrality indicators that are frequently used in SNA to assess the importance of nodes in the HSR network, such as degree centrality (DC), betweenness centrality (BC), and closeness centrality (CC) (Sheng et al. 2019; Zhang et al. 2020). Sheng et al. (2019) studied the nodes' topological importance in China's HSR network based on centrality measures, 
like DC, while using train frequency as a rough proxy of flow volume from a ticketing website serving about 704 HSR stations. Zhang et al. (2020) explored the impact of HSR construction on the urban agglomeration's spatial structure, and found that HSR construction has dramatically improved accessibility between cities, especially moderately developed cities.

A few studies explored high-speed railway network characteristics based on weighted network analysis. Jiao et al. (2017) used weighted centrality measures to evaluate cities' relative importance in a weighted HSR network, setting cities as nodes (instead of stations) and train frequency as the weight of the links. Chen et al. (2018) built an HSR weighted directed network and identified network accessibility changes based on certain indicators (node degree, strength, closeness, betweenness centrality) where weight represents the number of different HSR routes passing two stations.

Some studies concerned the development of HSR networks over the previous few years. Zhou and Qiu (2018) examined the evolution of HSR networks based on two periods: the development period between 2007 and 2017, and future construction plans for 2018 to 2030. They found that the Degree and Eccentricity of Tier 1 cities increased but PageRank decreased over time. Liu et al. (2020) studied the spatial disparities among Chinese cities based on HSR centrality indicator changes from 2010 to 2015 , and found that the disparity of accessibility was reduced with the expansion of a HSR network.

Many previous studies focused on the topological structure and evolution of high-speed railway networks. However, most of them used the unweighted network method, which cannot reflect edge information flow between two nodes. Some studies synthesized a weighted network (limited with unweighted centrality measures), or used train frequency as the weight, as shown in Table 1. Therefore, this study uses approximate transport capacity (seating capacity) to replace passenger flow between two HSR stations to obtain a weighted network. Then, we apply weighted centrality indicators (MA, TBC, and weighted harmonic centrality [WHC]) to evaluate the nodes' relative importance in the HSR network.

\subsection{Robustness of air transportation and high-speed rail networks}

Aside from SNA, air transportation and HSR networks have been analyzed as complex networks in the frame of graph theory in order to understand network robustness, which refers to the ability to avoid malfunctions when an emergency arises.

\subsubsection{Air transportation networks}

Some previous studies on the robustness of air transportation networks indicated that a small fraction of isolated critical nodes (airports, cities) would affect network connectivity significantly. Chi and Cai (2004) studied topological indicator changes in the US airport network and found that changes would reduce network efficiency by $25 \%$ if the top $10 \%$ of the most connected airports were removed. Lordan et al. (2014) presented a methodology for detecting critical airports in the global air transportation network, and measured network robustness using "size of giant component" when a network faced intentional attacks. They found that a small fraction of selected nodes, if isolated, could cause severe problems to the network's functioning. Kwon et al. (2019) evaluated major Asian airlines' network robustness using efficiency and the "size of a giant component". In addition, they based their study on airports' specific brokerage roles to explore hub nodes' impacts on network robustness.

\subsubsection{High-speed rail networks}

With the development of the HSR industry, HSR has become a common choice for travelers. Therefore, it would be inconvenient for travelers if train delays or cancellations due to emergencies happen. Zhang et al. (2016) assessed the structural vulnerability of high-speed railway networks subjected to malicious attacks in China, the US, and Japan, implementing Topological Efficiency and Unit Degree Betweenness as indicators. They concluded that network damage would happen if higher betweenness nodes were attacked. Feng et al. (2017) developed a comprehensive evaluation indicator to assess node importance, and simulated network vulnerability when stations were under random and malicious attacks based on different periods of time.

Li and Rong (2020) evaluated China's high-speed railway network robustness, especially the space-time failure effects of high-speed trains at typical HSR stations. But at the selected stations, they found the effects of train failure on the performance of the high-speed railway network were limited.

Based on the complex network theory, this study uses weighted network efficiency to evaluate the network robustness of HSR when a fraction of the nodes are removed. Meanwhile, we consider the link weight between the nodes, since weight can influence transportation efficiency, given the same path length (Zhou et al. 2019). Therefore, we use transport capacity to replace approximate passenger volume as the link weight. 
Table 1. Research on high-speed rail and air transportation networks by using weighted centrality

\begin{tabular}{|c|c|c|c|c|c|c|}
\hline Authors & Network & Nodes & Links & Link weight & $\begin{array}{l}\text { Unweighted centrality } \\
\text { measures }\end{array}$ & $\begin{array}{c}\text { Weighted } \\
\text { centrality measures }\end{array}$ \\
\hline $\begin{array}{l}\text { Jiao et al. } \\
(2017)\end{array}$ & HSR & Cities & Railway line & $\begin{array}{l}\text { Daily train } \\
\text { frequency }\end{array}$ & $\begin{array}{l}\text { Beta index, average path } \\
\text { length, and clustering } \\
\text { coefficient }\end{array}$ & $\begin{array}{l}\text { Weighted degree centrality, } \\
\text { weighted closeness } \\
\text { centrality, and weighted } \\
\text { betweenness centrality }\end{array}$ \\
\hline $\begin{array}{l}\text { Chen et al. } \\
(2018)\end{array}$ & HSR & Stations & Railway line & $\begin{array}{l}\text { Number of different } \\
\text { HSR routes }\end{array}$ & $\begin{array}{l}\text { Degree centrality, } \\
\text { closeness centrality, } \\
\text { and betweenness } \\
\text { centrality }\end{array}$ & Strength \\
\hline Sun et al. (2015) & Air & Airports & Direct flight & $\begin{array}{l}\text { Number of flights, } \\
\text { number of } \\
\text { available seats }\end{array}$ & $\begin{array}{l}\text { Degree centrality, and } \\
\quad \text { clustering coefficient }\end{array}$ & $\begin{array}{l}\text { Weighted degree, weighted } \\
\text { betweenness centrality, } \\
\text { and weighted closeness } \\
\text { centrality }\end{array}$ \\
\hline Du et al. (2017) & Air & Provinces & Direct flight & Number of flights & Average degree & Strength \\
\hline $\begin{array}{l}\text { Chung et al. } \\
(2020)\end{array}$ & Air & Airports & Direct flight & $\begin{array}{l}\text { Number of } \\
\text { passengers }\end{array}$ & $\begin{array}{l}\text { Degree centrality, } \\
\text { betweenness } \\
\text { centrality, pagerank, } \\
\text { and reverse pagerank }\end{array}$ & $\begin{array}{l}\text { Mean association, triangle } \\
\text { betweenness, weighted } \\
\text { pagerank, and reverse } \\
\text { weighted pagerank }\end{array}$ \\
\hline
\end{tabular}

HSR, high-speed rail.

Table 2. Research on the robustness of high-speed rail and air transportation networks

\begin{tabular}{|c|c|c|c|c|}
\hline Authors & Network & Nodes & Links & Robustness measures \\
\hline Zhang et al. (2016) & HSR & Stations & Railway lines & Network efficiency, connected diameter, and average unit degree betweenness \\
\hline Feng et al. (2017) & HSR & Stations & Railway lines & $\begin{array}{l}\text { Network efficiency, relative size of the largest connected subgraph, and the unit } \\
\text { degree betweenness }\end{array}$ \\
\hline $\mathrm{Gu}$ and $\mathrm{Li}(2019)$ & HSR & Cities & Railway lines & $\begin{array}{l}\text { Network efficiency, relative size of the largest connected subgraph, average } \\
\text { shortest path length, and average clustering coefficient }\end{array}$ \\
\hline $\begin{array}{l}\text { Wilkinson et al. } \\
\text { (2012) }\end{array}$ & Air & Airports & Airline routes & Degree distribution, and size of giant component \\
\hline Lordan et al. (2014) & Air & Airports & Airline routes & $\begin{array}{l}\text { Network efficiency, size of giant component, clustering coefficient, average } \\
\text { shortest path length, and average degree }\end{array}$ \\
\hline Du et al. (2016) & Air & Airports & Airline routes & Size of giant component \\
\hline Kwon et al. (2019) & Air & Airports & Airline routes & Network efficiency and size of giant component \\
\hline
\end{tabular}

HSR, high-speed rail.

\section{Methodology}

First, undirected unweighted networks were established based on whether there was an HSR or air transportation service between the cities, and unweighted centrality metrics (DC, node betweenness centrality [NBC], and harmonic centrality [HC]) were selected to measure the centrality of cities in terms of connections, intermediaries, and accessibility. Then, considering the passenger flow between cities as the link weight, HSR and air transport undirected weighted networks were synthesized and the corresponding weighted centrality measures (MA, TBC, and WHC) were applied to the weighted networks.

\subsection{Centrality analysis by weighted measures}

As the basic concept in SNA, centrality has been proposed to evaluate nodes' relative importance in a graph (De Nooy et al. 2018). Several metrics are used frequently in unweighted (binary) networks: like degree centrality, betweenness centrality, and closeness centrality (Freeman 1978; Nicosia et al. 2012; Opsahl et al. 2010). DC reflects the connection of the nodes, based on the sum of links incident upon a node; BC shows the probability of a node as a mediator in the network; CC reflects the relative reachability of nodes in the network, the sum of geodesic paths from a node to all other nodes (Nicosia et al. 2012). Some researchers also employed Eigenvector Centrality and PageRank to reflect the strong connections with these well-connected nodes (Pan et al. 2019; Wang and Cullinane 2016), or they used Subgroup Centrality to characterize the participation of each 
node in all subgraphs in a network (Estrada and Rodriguez-Velazquez 2005).

Because in unweighted (binary) networks these centrality measures cannot reflect information about the links, some researchers have proposed weighted centrality measures, which represent traffic dynamics in a weighted network (Barrat et al. 2004; Lee 2006; Opsahl et al. 2010).

Due to the limitations of CC, there is a lack of adjustability in networks with disconnected components, for example, if two nodes belong to different components but do not have a finite distance between them (Gao et al. 2013). So, this study uses HC rather than $\mathrm{CC}$, which can avoid cases where nodes are unreachable by other nodes, to evaluate accessibility. Therefore, DC, $\mathrm{BC}, \mathrm{HC}$, and the corresponding weighted centrality measures (MA, TBC, and WHC) were chosen as the centrality metrics. The weighted centrality measures are identified based on passenger traffic flow via HSR or air transportation between cities.

\subsubsection{Degree centrality}

DC as a "direct connection" measure shows the relative number of neighbors in the network connected to that node (Freeman 1978). The DC of nod $i$ is expressed as:

$$
D C(i)=\sum_{i \neq j \in V} \frac{x_{i j}}{N-1}
$$

where $x_{i j}$ is 1 if there is a link (edge) connecting nodes $i$ and $j$ ( 0 , otherwise), $V$ represents the set of all nodes in the network, and $N$ is the total number of nodes in the network. In this study, the number of different trains or flights passing through a specific city $i$ that has an HSR station or an airport is called the degree. Therefore, a city (with an HSR station or airport) with a higher DC has more direct connections to many other cities in the network.

\subsubsection{Mean association}

Lee (2006), based on node strength as proposed by Barrat et al. (2004), normalized MA to avoid the impact of node size. The MA of nod $i$ is expressed as:

$$
M A(i)=\sum_{i \neq j \in V} \frac{w_{i j}}{N-1}
$$

where $w_{i j}$ is the weight of the link between nodes $i$ and $j$. MA is used to measure the strength of each city (with a station or airport) in terms of the relative volume of passengers handled. A higher MA for a city indicates that more passengers associate it with other cities by HSR or air transport.

\subsubsection{Node betweenness centrality}

NBC shows the ability of a city (with a station or airport) to function as a mediator in the network. It is measured by the extent to which a node lies between all other pairs of nodes on their geodesic paths (Freeman 1978). The NBC of nod $i$ is expressed as:

$$
N B C(i)=\frac{2}{(N-1)(N-2)} \sum_{j \in V} \sum_{k \in V} \frac{g_{j k}(i)}{g_{j k}}
$$

where $i \neq j, j \neq k$, and $k \neq i, \quad g_{j k}$ is the number of geodesics linking nodes $j$ and $k$ and $g_{j k}(i)$ is the number of geodesics linking nodes $j$ and $k$ that contain node $i$, making $g_{j k}(i) / g_{j k}$ the probability that node $i$ acts as a mediator linking node $j$ and node $k$. The city with a higher NBC acts as the more important "intermediary" in HSR or air transportation networks.

\subsubsection{Triangle betweenness centrality}

NBC can only be used in a binary network. Therefore, Lee (2013) proposed TBC as a metric to measure "intermediaries" in 
weighted networks, replacing Weighted NBC proposed by Opsahl et al. (2010), which cannot evaluate when the geodesic between two nodes is also a direct path. The TBC of node $i$ can be formalized as follows:

$$
T B C(i)=\frac{2}{(N-1)(N-2)} \sum_{j \in V} \sum_{k \in V} f(i), f(i)=\left\{\begin{array}{c}
1, \text { if } \\
w_{j k}<\min \left(w_{i j}, w_{i k}\right) \\
0
\end{array}\right.
$$

If the volume flow from $j$ to $k$ through $i$ is greater than the flow from $j$ directly to $k$, the function $f(i)$ scores 1 for node $i$ (otherwise, 0 ) when there are three neighboring nodes $i, j, k$. In this study, a city with a higher TBC (including HSR stations or airports) acts as a transfer hub that maximizes the passenger traffic flow between the two other cities.

\subsubsection{Harmonic centrality}

$\mathrm{HC}$ was proposed by Marchiori and Latora (2000). It came from the idea of taking the harmonic mean of node-pair distances. $\mathrm{HC}$ of node $i$ is expressed as:

$$
H C(i)=\frac{1}{N-1} \sum_{i \neq j \in V} \frac{1}{d_{i j}}
$$

where $d_{i j}$ is the shortest distance between nodes $i$ and $j$. HC can avoid cases where some nodes are unreachable from other nodes, where an infinite distance outweighs the others: $d_{i j}=\infty$. HC is in the interval [0,1]. In this study, a higher HC score for a city that has HSR or airplanes passing by means that the city has better accessibility to other cities.

\subsubsection{Weighted harmonic centrality}

Based on HC proposed by Marchiori and Latora (2000), we established corresponding weighted centrality measure WHC of node $i$ as:

$$
W H C(i)=\frac{1}{N-1} \sum_{i \neq j \in V} \frac{1}{\sum_{l \in L_{i j}} 1 / w_{l}}
$$

where $w_{l}$ is the weight of link $l$, and $L_{i j}$ is the shortest path between $i$ and $j$. WHC in this study is used to measure accessibility in terms of the volume of passengers handled.

\subsection{Robustness analysis}

Network robustness refers to a system's ability to maintain its performance when facing disruptions (Zhou et al. 2017). In previous studies, there were many topological metrics used to assess network robustness, including the size of a giant component, average shortest path length, efficiency, etc. (Du et al. 2016; Kwon et al. 2019). In this study, WE is applied instead of efficiency to measure network robustness.

The efficiency proposed by Latora and Marchiori (2001) is a metric to measure the information exchange in networks that depends on the distance between node pairs. Efficiency is calculated from the inverse of the path distance between nodes. Based on this efficiency, Zhou et al. (2017), considering the effect of link weight on the efficiency of transmission, given the same path length, and proposed WE as the metric to measure the efficiency for a weighted network. WE of node $i$ can be formalized as follows:

$$
W E=\frac{1}{N(N-1)} \sum_{i \neq j \in V} e_{i j}=\frac{1}{N(N-1)} \sum_{i \neq j \in V} \frac{1}{\sum_{l \in L_{i j}} \frac{1}{W_{l}}}
$$

where $L_{i j}$ is the set of links along the shortest path between node pair $i$ and $j$ on the unweighted network, $l$ is the element in $L_{i j}$, and $w_{l}$ is the weight of link $l$. We repeatedly calculate the WE of networks when a fraction of the nodes are isolated, 
mainly by removing a fraction of the important nodes (from $1 \%$ to 10\%). The importance of nodes is determined by the weighted centrality ranking. And we then assess network robustness based on the changing trend of this WE.

\section{Data and network}

\subsection{Data}

\subsubsection{HSR data}

Since HSR passenger traffic data from China is not easy to collect, this study uses monthly data on HSR seating capacity instead of approximate passenger traffic.

The HSR data were mainly collected from the Chinese railway ticketing website and from information on passenger train information. The ticketing website (www.12306.cn) contains information on all existing HSR trains, including routes, stations, fares, etc., excluding Hong Kong, Macau, and Taiwan. At the same time, the HSR information on passenger train formation includes carriage types, seating capacity, etc.

This study selected only data with a monthly frequency of more than 10 and regarded it as fixed-route data, excluding additional trains during the peak holiday period. As done in previous studies (Cao et al. 2019), we treated cities as nodes instead of using HSR stations. All HSR stations in the same city are attributed to that city. For example, the Beijing, Beijing West, and Beijing South railway stations are all assigned to the node City Beijing. If there is an HSR train service between city $i$ and city $j$, the two cities are connected. Totals include 197 cities and 7,966 railways, which comprised 3,287 G-trains, 3,032 D-trains, and 1,647 C-trains.

\subsubsection{Air transportation data}

The passenger data from airports were collected from statistical data on civil aviation in China for 2018: "passenger traffic on major domestic origin-destination pair (OD) markets.". As with previous studies (Liu et al. 2018), this study aggregates airport passenger traffic on major domestic OD markets as domestic passenger traffic.

\subsection{Network properties}

The analysis focused on the city network, not the high-speed railway stations or airports, setting origins and final destinations for pairs of cities traveled by passengers as the nodes and links. In graph $G=(V, E)$, the node (vertex) set, $V$, represents cities, and the link (edge) set, $E$, represents passenger movement between cities via HSR or airplane.

An adjacency matrix, $X_{n \times n}$, represents the connections as $X_{i j}=1$ when more than one passenger moves between cities $i$ and $j ; X_{i j}=0$, otherwise. A weighted adjacency matrix, $W_{n \times n}$, is created where $W_{i}$ represents the link weight for the number of passengers moving between cities $i$ and $j$ via HSR or airplane. The numbers of nodes and links are shown in Table 3.

\section{Network analysis}

\subsection{Centrality analysis}

Centrality reflects the relative importance of each node (city) in the HSR network. For a city with a higher centrality ranking, it is easier to access resources and information, and it has greater power and influence in the network. Three unweighted centrality measures and their corresponding weighted centrality measures were selected for analysis.

To better understand the spatial distributions of different levels of cities and the hierarchical structure of China's HSR network, the centrality results are divided into six levels by their natural breaks (called the Jenks). This method of dividing classes is based on natural groupings inherent in the data. Furthermore, the spatial distribution and classification results of HSR

Table 3. Network characteristics

\begin{tabular}{ccc}
\hline Property & HSR & Air \\
Number of nodes & 197 & 73 \\
Number of links & 7,966 & 376 \\
\hline
\end{tabular}

HSR, high-speed rail. 
Table 4. Spatial distribution characteristics of the HSR network

\begin{tabular}{|c|c|c|c|}
\hline & City nodes & Stations & Cities in these provinces \\
\hline Eastern & 69 & 129 & $\begin{array}{l}\text { Beijing, Tianjin, Hebei, Shanghai, Jiangsu, Zhejiang, Fujian, Shandong, } \\
\text { Guangdong, and Hainan }\end{array}$ \\
\hline Central & 52 & 77 & Shanxi, Anhui, Jiangxi, Henan, Hubei, and Hunan \\
\hline Western & 57 & 104 & $\begin{array}{l}\text { Inner Mongolia, Guangxi, Chongqing, Sichuan, Guizhou, Yunnan, Tibet, } \\
\text { Shaanxi, Gansu, Qinghai, Ningxia, and Xinjiang }\end{array}$ \\
\hline Northeast & 19 & 35 & Liaoning, Jilin, and Heilongjiang \\
\hline
\end{tabular}

cities were visualized by using ArcGIS 10.2.

According to the statistical caliber of the four economic regions of the National Bureau of Statistics, a city is designated as belonging to one of four regions: East, Central, West, and Northeast (Yu et al. 2013). From the spatial distribution characteristics of HSR cities, as shown in Table 4, there are 69 cities in the Eastern region (35\% of the total); the Central Region has 52 cities (26\%); in the Western Region are 57 cities (29\%); and the Northeast region has 19 cities (10\% of the total). The spatial distribution shows there are more cities with HSR stations in the Eastern region.

\subsubsection{Comparison between unweighted and weighted centrality measures}

If a network is a weighted network, it would be more effective to analyze the network's characteristics using weighted centrality measures than unweighted centrality measures (Chung et al. 2020). Following the process in previous studies, the results of unweighted and weighted centrality are compared in Figure 1.

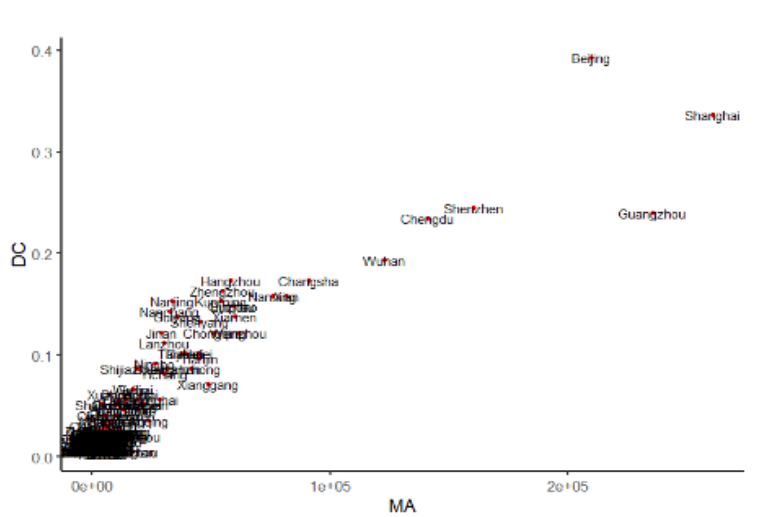

(a) MA and DC

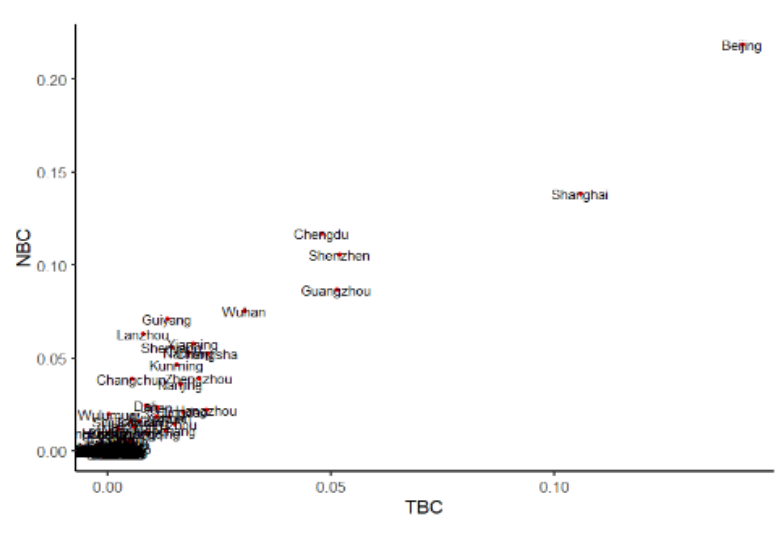

(b) TBC and NBC

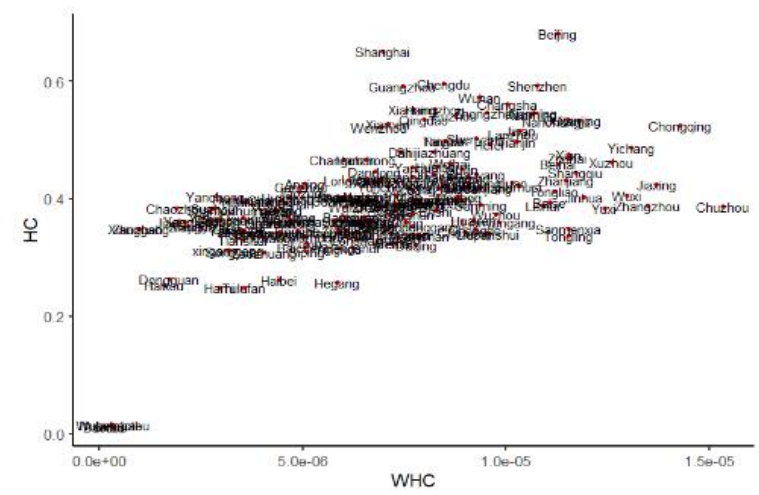

(c) HC and WHC

Figure 1. Scatter plot showing unweighted and weighted centrality. MA, mean association; DC, degree centrality; TBC, triangle betweenness centrality; NBC, node betweenness centrality; HC, harmonic centrality; WHC, weighted harmonic centrality. 
Beijing, Shanghai, and Guangzhou show the highest DC, but the highest MA rankings are for Shanghai, Guangzhou, and Beijing. This indicates that a city (with HSR stations) with many connections is not necessarily associated with many passengers. Besides, NBC and TBC are metrics to measure nodes as mediators. Except for Beijing and Shanghai, most of the cities' rankings differ in NBC and TBC. This may be because Beijing and Shanghai are China's political and economic centers, respectively; they are both intermediaries in the HSR network, and they act as transport hubs that maximize passenger traffic flow between two other cities.

Based on HC proposed by Marchiori and Latora (2000), the corresponding weighted centrality measure WHC was applied in this study to measure accessibility considering passenger volume. The result shows a considerable difference in node rankings when comparing $\mathrm{HC}$ and WHC. Above all, weighted centrality measures are more useful indicators in a weighted network. Therefore, in the following sections, we investigate network characteristics based on weighted centrality measures.

\subsubsection{Comparison between different weighted centrality measures}

A huge difference can be found between different weighted centrality results shown in the Figure 2 and Table 5. MA shows the strength of the connection between cities, where strength reflects passenger flow. Shanghai, Guangzhou, and Beijing are in the top three. Simultaneously, there is a considerable gap between these three cities and other cities. From Figure 3, the spatial distribution of the centrality results indicates that more intensive cities with HSR stations are concentrated in the Yangtze River Delta and the Pearl River Delta, which may be due to frequent HSR service and large amounts of passenger traffic on the Guang - Shen intercity rail (Guangzhou to Shenzhen), the Jing - Hu HSR (Beijing to Shanghai) and the Hu - Han - Rong HSR

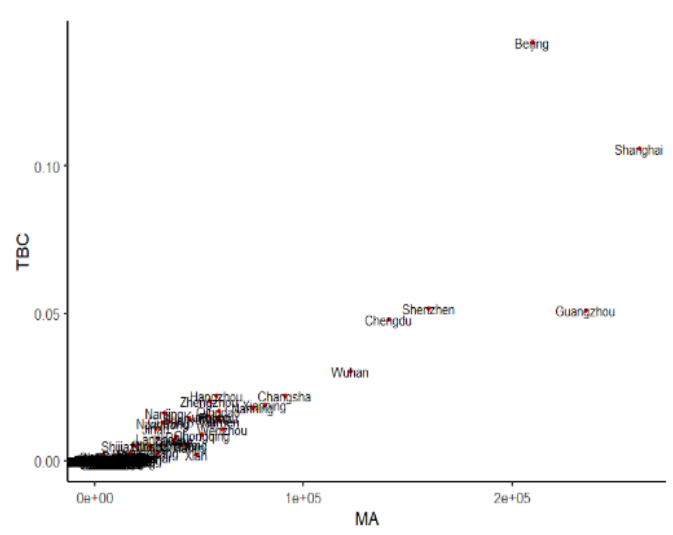

(a) MA and TBC

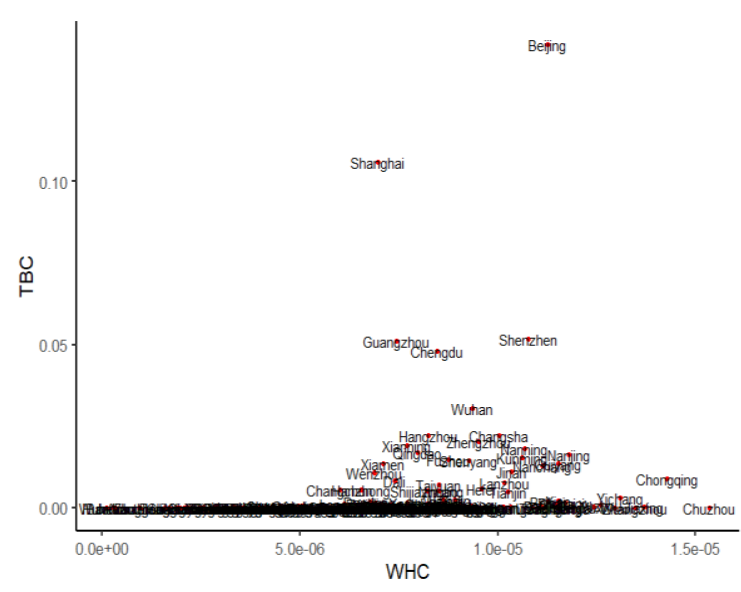

(c) WHC and TBC

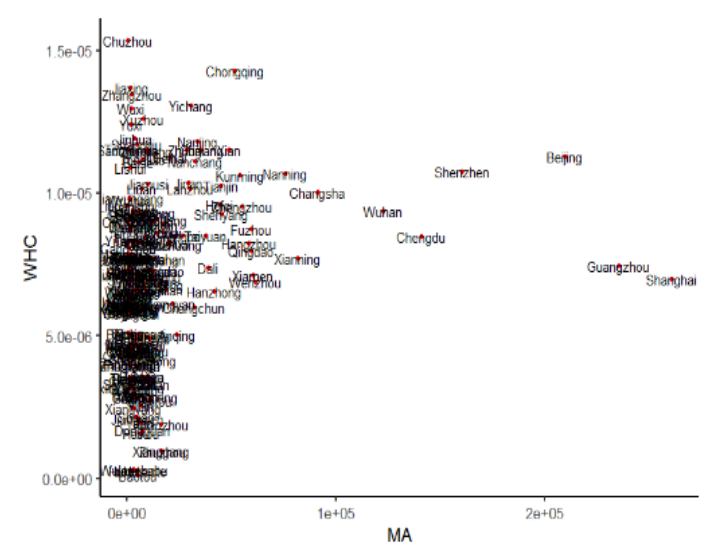

(b) MA and WHC

Figure 2. Scatter plot of weighted centralities. MA, mean association; TBC, triangle betweenness centrality; WHC, weighted harmonic centrality. 
(Shanghai - Nanjing - Hefei - Wuhan - Chongqing - Chengdu). Among the top three cities with the highest MA rankings, Shanghai is the economic center of China and an important transportation center in Asia, and Beijing is the political center of China. Also, Guangzhou is the center of China's southern coastal area, which strongly influences the HSR network. Besides, Nanning, Wenzhou, Xiamen, and Chongqing have much higher MA rankings than TBC, indicating that many passengers are associated with these cities, but these cities do not act as transport hubs.

The larger the TBC, the stronger the load capacity of traffic flow between cities. TBC shows that a city acts as a transit center that mediates two other neighboring cities when there are more passengers to go through the cities. These cities are hubs that play an important role in controlling traffic flow and increasing efficiency for travelers who transfer. Therefore, the railway administration should pay attention to the safety and protection of HSR stations in these cities. Shanghai has the highest MA ranking, while Beijing has the highest TBC ranking, indicating Beijing is the dominant intermediary city in the HSR network. More passengers travel from one city to another by high-speed train and transfer in Beijing. That may be because Beijing is the political and transportation center of China, and it functions as a major hub in the domestic transport network. So Beijing should give full play to its role of radiating diffusion to surrounding cities.

The cities with larger TBC are mostly provincial capitals or regional centers, including Beijing, Shanghai, Shenzhen, Guangzhou, Chengdu, Wuhan, Changsha, Hangzhou, Zhengzhou, Xi'an, etc. These cities occupy essential positions in the HSR network, and they are also intermediaries and bridges for many passengers who transfer to other cities. Besides, the cities with lower TBC may be mainly affected by the radiating role of the above cities. However, the TBC of 85 cities in the HSR network

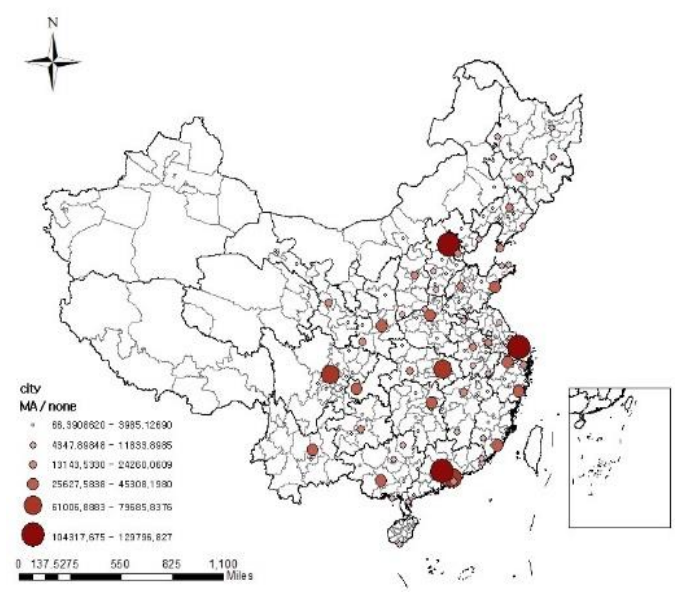

(a) MA

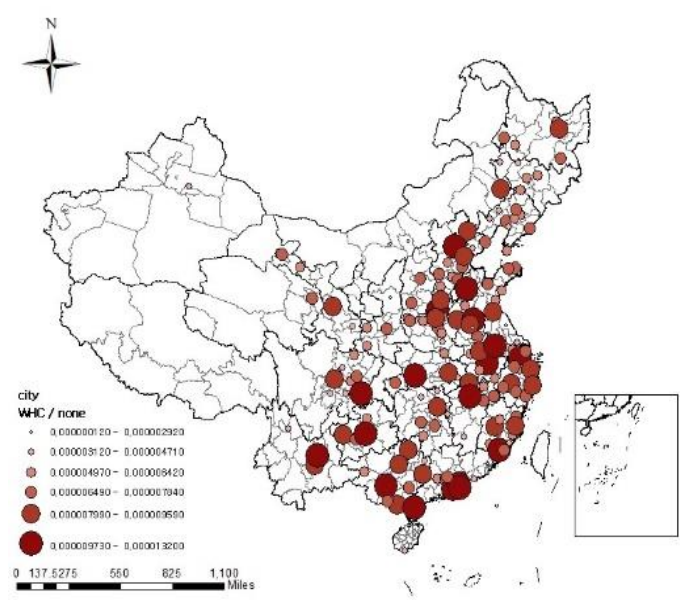

(c) WHC

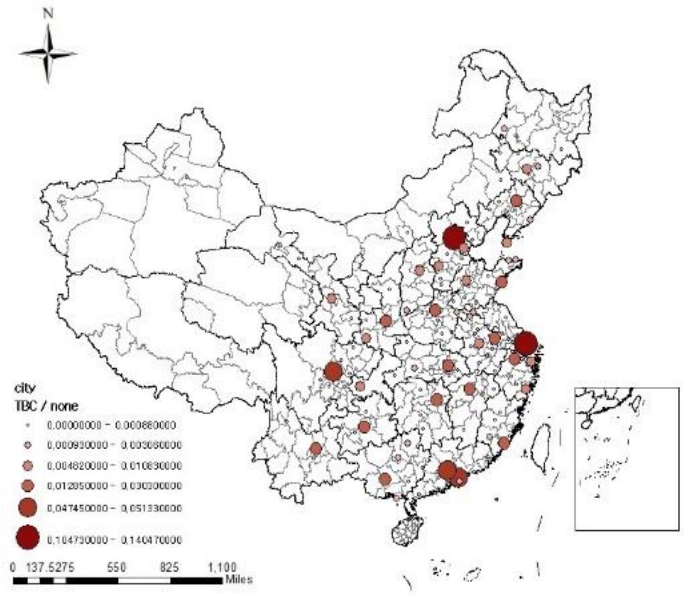

(b) TBC

Figure 3. The spatial distribution of city nodes in the HSR network. MA, mean association; TBC, triangle betweenness centrality; WHC, weighted harmonic centrality. 
Table 5. Centrality analysis results of city nodes in the HSR network

\begin{tabular}{|c|c|c|c|c|c|c|}
\hline Rank & Node & MA & Node & TBC & Node & WHC \\
\hline 1 & Shanghai & $260,918.1122$ & Beijing & 0.14192 & Chuzhou & 0.000015342 \\
\hline 2 & Guangzhou & $235,426.6735$ & Shanghai & 0.10581 & Chongqing & 0.000014285 \\
\hline 3 & Beijing & $209,699.8163$ & Shenzhen & 0.05186 & Jiaxing & 0.000013696 \\
\hline 4 & Shenzhen & $160,184.7959$ & Guangzhou & 0.05123 & Zhangzhou & 0.000013474 \\
\hline 5 & Chengdu & $140,806.1735$ & Chengdu & 0.04793 & Yichang & 0.000013083 \\
\hline 6 & Wuhan & $122,636.2959$ & Wuhan & 0.03061 & Wuxi & 0.000012968 \\
\hline 7 & Changsha & $91,078.72449$ & Changsha & 0.02219 & Xuzhou & 0.000012607 \\
\hline 8 & Xianning & $81,736.0102$ & Hangzhou & 0.02203 & Yuxi & 0.000012420 \\
\hline 9 & Nanning & $75,771.43878$ & Zhengzhou & 0.0203 & Jinhua & 0.000011915 \\
\hline 10 & Wenzhou & $61,422.19388$ & Xianning & 0.01915 & Nanjing & 0.000011799 \\
\hline 11 & Xiamen & $60,192.54082$ & Nanning & 0.01816 & Shangqiu & 0.000011706 \\
\hline 12 & Fuzhou & $59,416.40816$ & Qingdao & 0.01685 & Tongling & 0.000011533 \\
\hline 13 & Qingdao & $59,271.41837$ & Nanjing & 0.01627 & Sanmenxia & 0.000011531 \\
\hline 14 & Hangzhou & $58,175.16327$ & Kunming & 0.01533 & Xian & 0.000011531 \\
\hline 15 & Zhengzhou & $54,895.71429$ & Fuzhou & 0.01491 & Guiyang & 0.000011522 \\
\hline 16 & Kunming & $53,817.13265$ & Shenyang & 0.01439 & Zhuhai & 0.000011515 \\
\hline 17 & Chongqing & $51,516.67347$ & Xiamen & 0.01366 & Zhanjiang & 0.000011468 \\
\hline 18 & Xian & $48,767.67347$ & Guiyang & 0.0134 & Beijing & 0.000011268 \\
\hline 19 & Shenyang & $45,178.61224$ & Nanchang & 0.01298 & Beihai & 0.000011263 \\
\hline 20 & Tianjin & $44,755.07143$ & Jinan & 0.01094 & Tongliao & 0.000011175 \\
\hline
\end{tabular}

MA, mean association; TBC, triangle betweenness centrality; WHC, weighted harmonic centrality.

is 0 , showing that the intermediary capabilities of cities are significantly different, and that HSR network development is uneven.

At the same time, some cities, such as Chongqing and Xi'an, have much higher MA and WHC rankings than TBC, indicating that if a city has many HSR passengers and high accessibility, that does not mean its intermediary ability is strong.

HC comes from the idea of taking the harmonic mean of the node-pair distances (Marchiori and Latora 2000; Liu et al. 2018), which indicates a kind of accessibility. The higher the HC, the better the city's accessibility. We accordingly propose WHC based on it, considering passenger volume between cities as the link weight.

The WHC rankings are somewhat different from other weighted centrality results. A city with a higher WHC has better accessibility to other cities, such as Chuzhou, Chongqing, and Jiaxing. For other cities, there are noticeable regional differences. The accessibility of eastern and central cities is significantly better than western and northeast cities. Besides, the 10 cities with the worst accessibility are located in the northeast, northwest, and southwest of China.

\subsubsection{Comparison between the weighted centrality of HSR and air}

MA reflects the strength of the connection between cities, based on the passenger flow between cities by air and HSR. Figure 4 shows the scatter plots of weighted centrality scores between HSR and air transportation network for the top 20 cities. Shanghai, Guangzhou, and Beijing have the highest MA in the HSR network, but their air MA rankings change to Shanghai, Beijing, and then Guangzhou. There is a huge difference in MA results between HSR and air transportation networks. For instance, Shenzhen, Wuhan, and Changsha have a higher MA in the HSR network, but a lower MA in the air transportation network. In contrast, Kunming, Hangzhou, Chongqing, and Hangzhou have a lower MA in the HSR network, but their air MA rankings are higher.

Triangle betweenness centrality represents the ability of a node to be a mediator in a weighted network. A city with a higher TBC is a transfer hub that mediates two other neighboring cities when more passengers choose to go through those cities by HSR or airplane. In the HSR network, the TBC of Shenzhen ranked third, but Shenzhen ranked ninth in the air transportation network. In addition, Wuhan and Qingdao have a lower ranking in the air transportation network than the HSR network. On the other hand, some cities, such as Xi'an and Kunming, have a higher TBC in the air transportation network, but ranking decreases a lot in the HSR network. The TBC ranking of Kunming has changed dramatically, decreasing from fourth to fourteenth. 


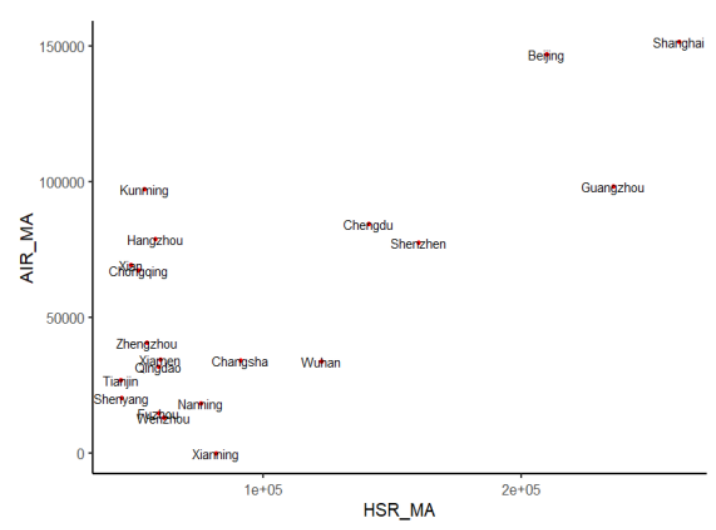

(a) MA: HSR and Air

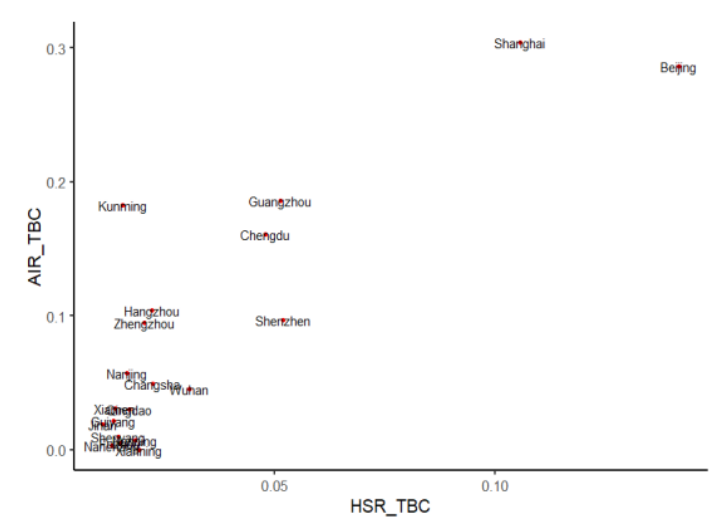

(b) TBC: HSR and Air

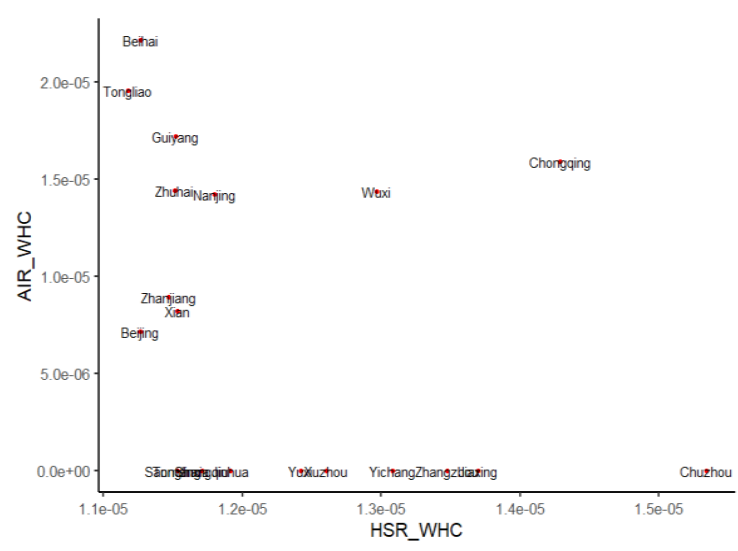

(c) WHC: HSR and Air

Figure 4. Scatter plot between HSR and air for the Top 20 cities. MA, mean association; HSR, high-speed rail; TBC, triangle betweenness centrality; WHC, weighted harmonic centrality.

The difference in centrality results between HSR and air transportation networks shows obvious spatial differentiation characteristics, as shown in Figure 5. The level of economic development in eastern cities is higher than in western ones. There is not much difference in the ranking of cities' centrality between HSR and air transportation in eastern cities, where many cities have higher air rankings and HSR rankings. In the central region, many cities have complementary roles in HSR and air transportation networks. For example, Wuhan, Changsha, and Xianning have higher HSR rankings than air rankings. However, air transportation is still dominant in western cities, except for some big cities, such as Chengdu, Chongqing, and Xi'an. Many cities have higher centrality rankings in the air transportation network than in the HSR network. The difference may be due to the location and lag effect of HSR development.

The cities with higher air and HSR rankings should seize the opportunity for economic development, and other cities need to pay attention to the potential impact of these cities. Moreover, owing to the huge gap in centrality results between HSR and air transportation networks in western cities, the central government should accelerate the construction and extension of highspeed railway lines to the western region to close the gap. And the local government can strengthen the cooperation between the two transportation modes to provide people with convenient and efficient travel services.

\section{Network robustness analysis}

The network's overall structure and connectivity will be influenced by the removal of a fraction of the nodes. Many studies have focused on network robustness, which refers to the ability to avoid network malfunctions when a fraction of nodes or links are damaged (Albert et al. 2000), so it is closely related to network efficiency. Now, robustness analysis is being used in transportation network analysis for air, maritime, railway, and urban transportation networks (Liu et al. 2018; Lordan et al. 2016). 
Table 6. Top 10 centrality rankings of city nodes in HSR and air transportation networks

\begin{tabular}{|c|c|c|c|c|c|c|}
\hline \multirow{2}{*}{ Rank } & \multicolumn{3}{|c|}{ HSR } & \multicolumn{3}{|c|}{ Air } \\
\hline & MA & TBC & WHC & MA & TBC & WHC \\
\hline 1 & Shanghai & Beijing & Chuzhou & Shanghai & Shanghai & Enshi \\
\hline 2 & Guangzhou & Shanghai & Chongqing & Beijing & Beijing & Hailaer \\
\hline 3 & Beijing & Shenzhen & Jiaxing & Guangzhou & Guangzhou & Mianyang \\
\hline 4 & Shenzhen & Guangzhou & Zhangzhou & Kunming & Kunming & Beihai \\
\hline 5 & Chengdu & Chengdu & Yichang & Chengdu & Chengdu & Zhangjiajie \\
\hline 6 & Wuhan & Wuhan & Wuxi & Hangzhou & Chongqing & Jiamusi \\
\hline 7 & Changsha & Changsha & Xuzhou & Shenzhen & Xi'an & Huhehaote \\
\hline 8 & Xianning & Hangzhou & Yuxi & Xi'an & Hangzhou & Fuzhou \\
\hline 9 & Nanning & Zhengzhou & Jinhua & Chongqing & Shenzhen & Tongliao \\
\hline 10 & Wenzhou & Xianning & Nanjing & Zhengzhou & Zhengzhou & Xilinhaote \\
\hline
\end{tabular}

HSR, high-speed rail; MA, mean association; TBC, triangle betweenness centrality; WHC, weighted harmonic centrality.

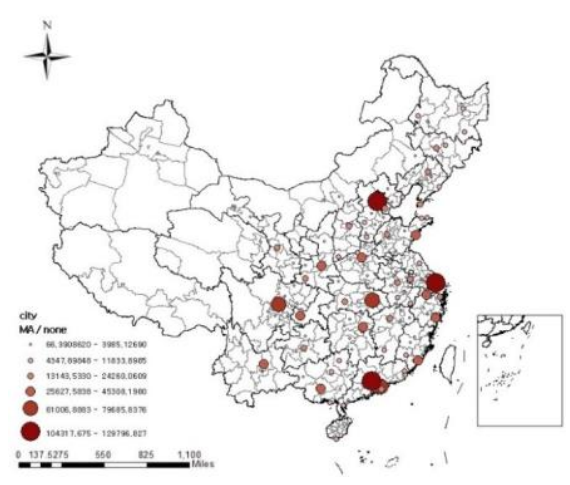

(a) MA: HSR network

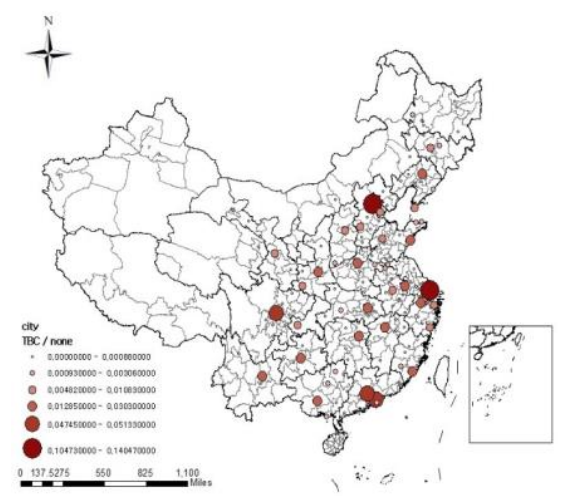

(c) TBC: HSR network

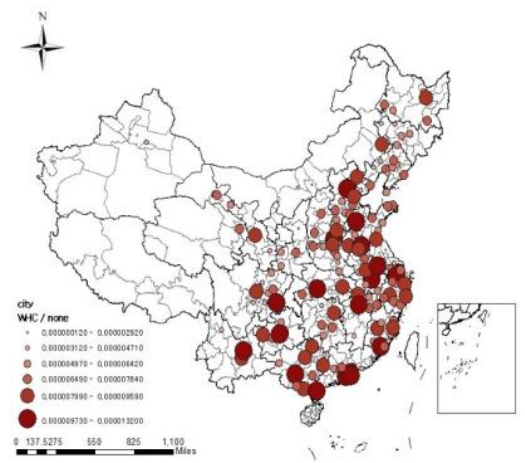

(e) WHC: HSR network

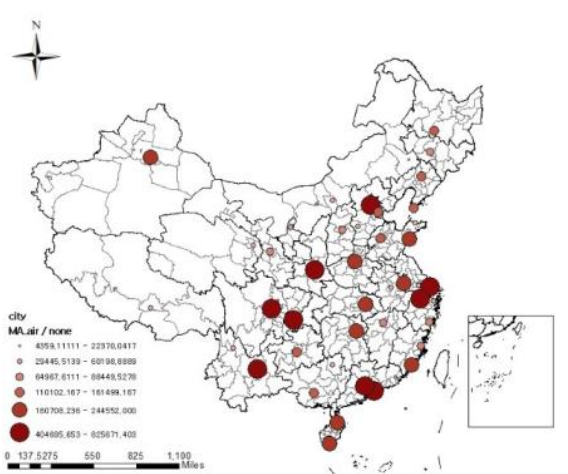

(b) MA: Air transportation network

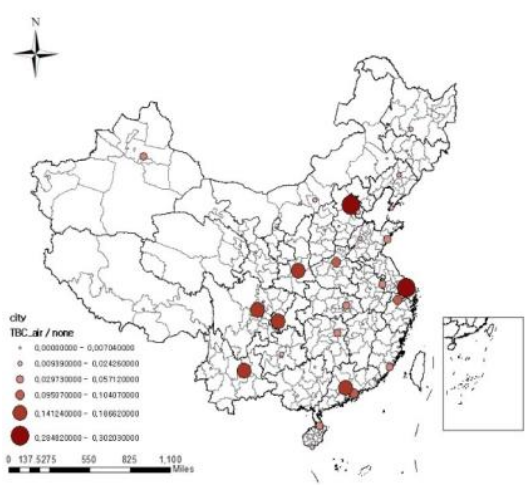

(d) TBC: Air transportation network

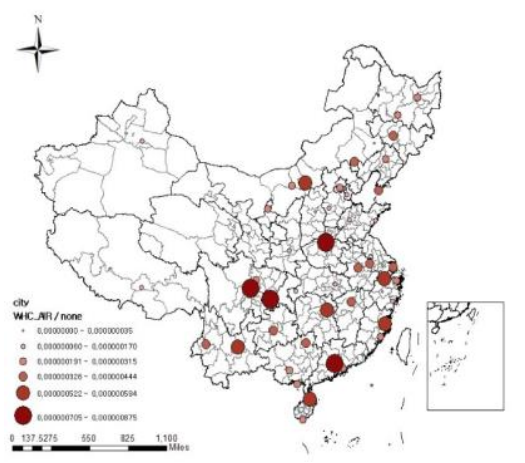

(f) WHC: Air transportation network

Figure 5. Spatial distribution of city nodes in HSR and air transportation networks. MA, mean association; HSR, high-speed rail; TBC, triangle betweenness centrality; WHC, weighted harmonic centrality. 
In this study, network robustness is the ability to maintain network connectivity when some nodes (cities) and links (HSR lines or flight routes) are interrupted by emergency events, such as high-speed train or flight delays or cancellations due to extreme weather, natural disasters, or epidemics. The network robustness of HSR, air, and multimodal networks are analyzed by measuring WE when some of the nodes have been removed and according to weighted centrality rankings (MA, TBC, and WHC).

\subsection{Multimodal network configuration}

A multimodal network is constructed by superimposing HSR and air transportation networks, and cities are treated as nodes, instead of stations or airports. If there is a non-stop HSR train or airline service between city $i$ and city $j$, the two cities are regarded as connected.

In the graph $G=(V, E)$, the node (vertex) set, $V$, represents cities, and the link (edge) set, $E$, represents passenger movement between cities by airplane or HSR. There are three types of nodes, $V=\left(V^{\text {air }}, V^{\text {HSR }}, V^{\text {air-HSR }}\right)$, which are cities with airports, cities with HSR stations, and cities with both airports and HSR stations, respectively. From the perspective of spatial location, most cities with both airports and HSR stations are large cities concentrated in the east of China.

A weighted adjacency matrix, $W n \times n$, is created where $w i j$ represents the link weight. Considering that the speed of transportation may affect people's choice of travel mode, we use the passenger volume between city $i$ and city $j$ multiplied by the speed ratio to obtain the link weight. When there is an overlap between air and HSR, such as City A and City B in Figure 6 (two cities connected with air and HSR), suppose the link weight is the sum of their corresponding weights. For a total of 218 city nodes, 8,342 links are included in the multimodal network.

$$
\begin{aligned}
& \text { weight }_{i j}^{A}=\operatorname{pax}_{i j}^{A} * \frac{\text { speed }^{A}}{\text { speed }^{A}+\text { speed }^{H}} \\
& \text { weight }_{i j}^{H}=\operatorname{pax}_{i j}^{H} * \frac{\text { speed }^{H}}{\text { speed }^{A}+\text { speed }^{H}}
\end{aligned}
$$

\subsection{Robustness analysis}

\subsubsection{Robustness in air transportation and HSR networks}

This study measured the network robustness of the air transportation and HSR networks in China using WE when isolation of a fraction of the nodes increases from $1 \%$ to $10 \%$, where MA, TBC, and WHC are used as the criteria for node isolation. Robustness analysis results of air transportation and HSR networks are shown in Figure 7.

When $1 \%$ of well-connected nodes become isolated from the air transportation network, the WE decreases by $2.3 \%, 2.4 \%$, and $81.6 \%$ for MA, TBC, and WHC, respectively. However, in the HSR network, the WE decreases only slightly, by $0.63 \%$, $2.3 \%$, and $13.7 \%$, respectively. That shows that as the fraction of nodes in isolation increases, WE decreases more in the air transportation network than in the HSR network.

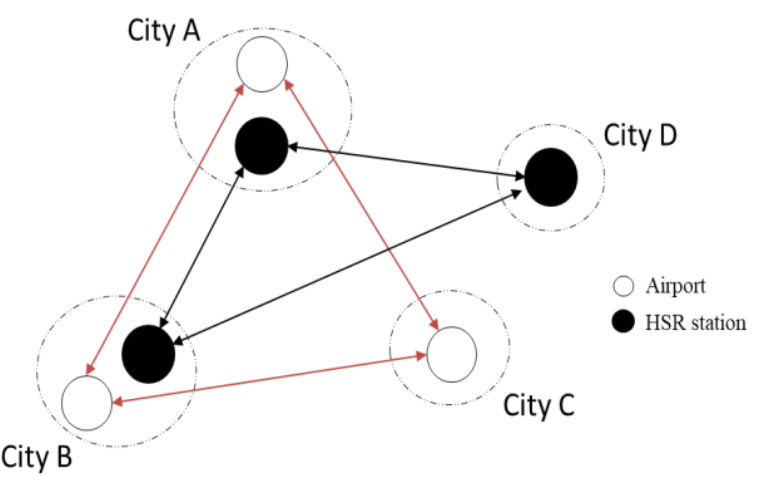

Figure 6. The multimodal network. HSR, high-speed rail. 
When $10 \%$ of nodes are isolated, WE decreases by nearly $50 \%$ in the air transportation network for MA and TBC. At the same time, it decreases by $38.8 \%$ and $44.7 \%$ for MA and TBC, respectively, in the HSR network. This shows that an HSR network has relatively higher network robustness than an air transportation network. When the service of well-connected nodes (cities) and links (HSR lines or flight routes) is disrupted by an emergency, air transportation network robustness decreases more quickly than the HSR network. Passengers maybe cannot arrive in some cities or must spend more time transferring to other cities by plane.

It may be because air transportation networks are usually scale-free, in which a few hub airports directly connect to most other airports, and most airports have few connections to the others (Barabási and Albert 1999). Therefore, in some critical airports, the air transportation network is more vulnerable to service interruptions (Zhou et al. 2019). If some HSR stations break down, however, there still are alternative routes and trains to get passengers to their destinations.

In this simulation, from node isolation based on nodes with the highest centrality scores to nodes with the lowest scores, we can see that isolation of well-connected nodes will rapidly lead to structure and connectivity change, resulting in network breakdown. Therefore, determining critical nodes in the network can help to increase reliability. On one hand, managers should pay more attention to these airports or HSR stations, and add more security inspections at critical periods, or a failure may affect many passengers' travel plans. That will also facilitate developing contingency plans for critical nodes, such as rescheduling flights or trains to recover from disruptions (Cadarso et al. 2013). On the other hand, public transportation stations are likely to become vectors of a virus, resulting in the occurrence of a cluster of infections or a "super-spreading" event (Shen et al. 2020). So if the government or related firms can control passenger flow or block critical nodes, they can slow down or stop the spread of epidemics such as COVID-19.

\subsubsection{Robustness of the multimodal network}

Multimodal network robustness has been investigated by measuring WE when some of the nodes (cities with airports or HSR stations) fail. In this simulation, the isolated nodes accounted for $1 \%$ to $10 \%$ of the highest and lowest centrality scores.

For example, in Figure 6, if the HSR station of City A (with both an HSR station and an airport) is paralyzed, passengers can still travel from City A to City B by airplane instead of by HSR. Figure 8 shows WE changes to MA, TBC, and WHC in a multimodal network when some cities with HSR stations or airports in isolation are subjected to the highest MA, TBC, and WHC node-based attacks. In the first iteration, WE decreased by 1.0\%, 1.1\%, and 35.1\% for cities with HSR stations or airports that were paralyzed when the highest MA, TBC, and WHC nodes, respectively, were isolated. It shows that WE gradually decreases when the fraction of nodes in isolation increases in nodes with the highest centrality. Therefore, the multimodal network has a good ability to maintain connectivity in response to emergencies such as extreme weather, natural disasters, etc., when it comes to HSR or airports in operation.
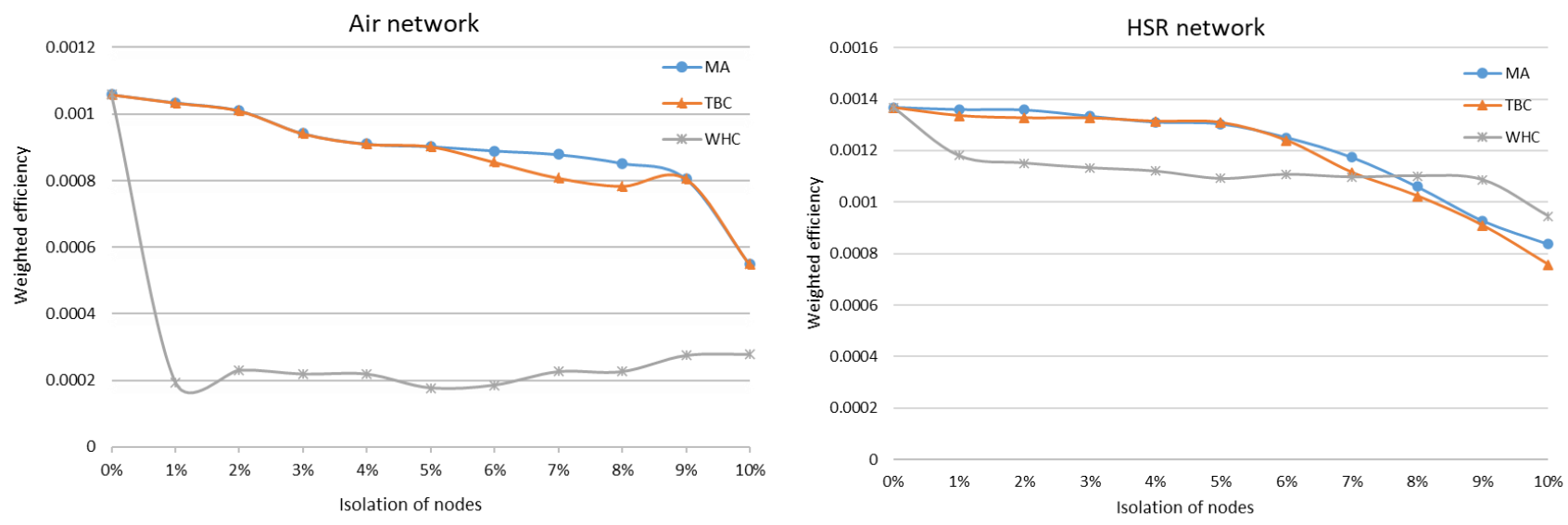

Figure 7. Air and HSR network robustness analysis results: weighted efficiency. MA, mean association; TBC, triangle betweenness centrality; WHC, weighted harmonic centrality; HSR, high-speed rail. 


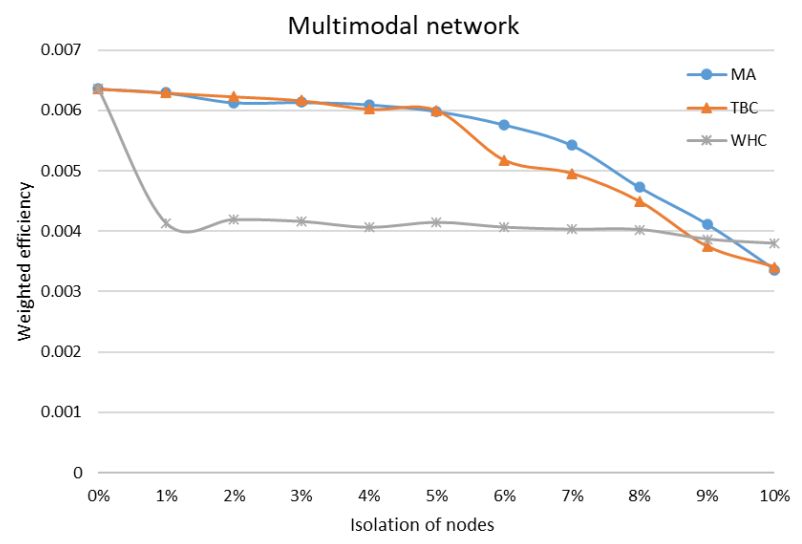

Figure 8. Multimodal network robustness analysis results: weighted efficiency. MA, mean association; TBC, triangle betweenness centrality; WHC, weighted harmonic centrality.

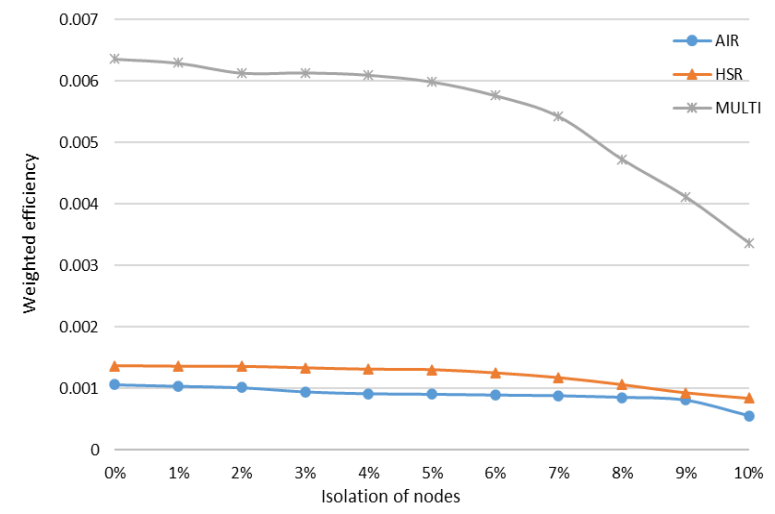

(a) MA node-based isolation

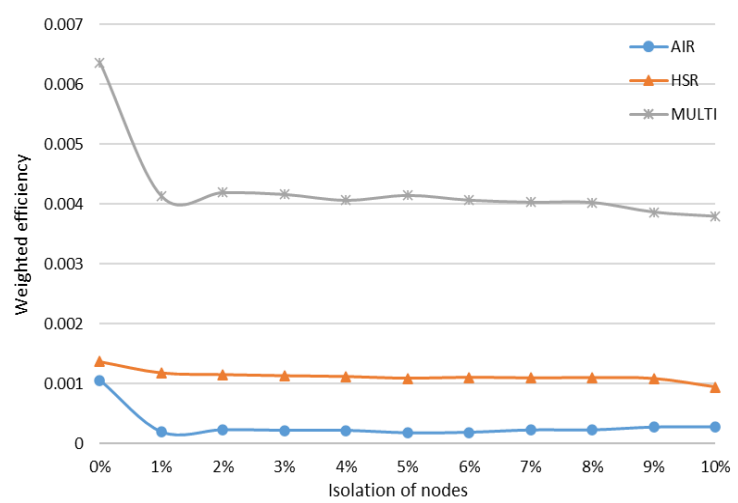

(c) WHC node-based isolation

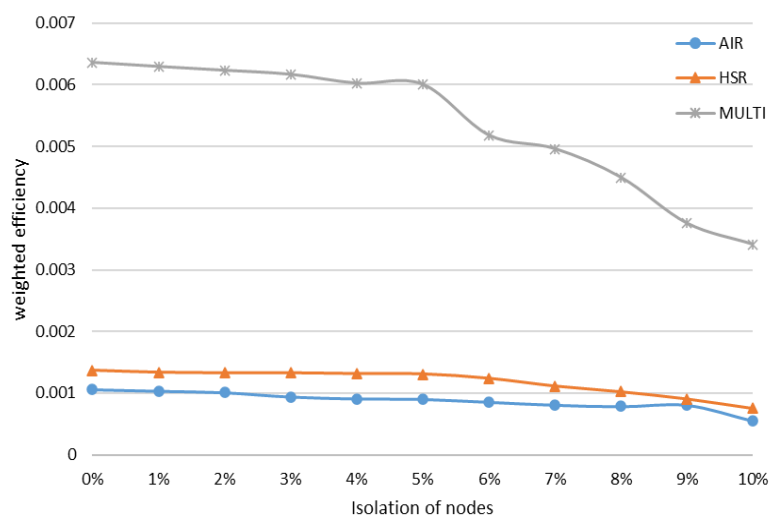

(b) TBC node-based isolation

Figure 9. Robustness analysis results of three types of network. MA, mean association; TBC, triangle betweenness centrality; WHC, weighted harmonic centrality.

Figure 9 compares network robustness of three transportation networks (air, HSR, and multimodal networks) with each other. By comparing these three networks, we found that robustness was different. The results clearly show that a multimodal network (airports and high-speed stations) has the best ability to maintain connectivity, whether compared to HSR-only or air transportation-only networks. As the number of failed HSR stations and airports increases, we found the WE of the multimodal network was still much higher than air and HSR networks. The single HSR or air transportation network is more likely to be 
affected by well-connected nodes becoming isolated, since there are fewer links that connect the isolated stations or airports to others. But in the multimodal network, if a fraction of the airports or HSR stations has become paralyzed, people could still choose alternative transportation modes.

Usually, HSR stations in hub airports can provide a feeder service for air demand, since travelers now have two fast alternatives offering high-frequency services for their journeys (Albalate et al. 2015; Givoni and Banister 2006). In particular, air-HSR multimodal services could alleviate capacity pressure to improve social welfare if hub airport capacity is significantly constrained or falls under a disruptive event (Chen and Rose 2018; Jiang and Zhang 2014). Therefore, the air-HSR multimodal network has been generally perceived as a critical solution to improving network robustness to emergencies, such as extreme weather (Zhou and Chen 2020). It can provide people with an alternative travel mode despite disruptive events.

At the same time, HSR and aviation networks have the space for coordinated development, which reflects the superiority of coordinated development of multimodal transportation. Local government and related firms can strengthen cooperation between the two transportation modes to improve robustness against failures. While the success of air-HSR cooperation also partially depends on whether the rail service arrives to meet banks of connecting flights, and whether rail stations are located at the airport (Clewlow et al. 2012), HSR construction needs to be well considered.

\section{Conclusion}

This study explored the characteristics of HSR and air transportation networks in China based on the weighted complex network approach. This study identified which node plays a central role in HSR and air transportation networks through weighted centrality analysis. Through robustness analysis, we analyzed how vulnerable the transportation network can be when an unexpected attack or isolation occurs in nodes with high centrality.

Several weighted centrality measures (MA, TBC, and WHC) were applied to reflect the information of links such as passenger traffic or capacity and to perform a more precise analysis of the network characteristics. In addition, the spatial distribution of centrality results was visualized by using ArcGIS 10.2. Moreover, we synthesized multimodal networks (including both airports and HSR stations), treating cities as nodes and regarding two cities as connected if there is a non-stop HSR train service or airline between them. Then, WE was used as an indicator to analyze the network robustness of HSR, air, and multimodal networks when a fraction of well-connected nodes became isolated.

First, from the city nodes' spatial distribution based on weighted centrality results in the HSR network, we found that cities with a higher MA are concentrated in the Yangtze River Delta and the Pearl River Delta. That indicated that there are many passengers associated with these cities. Moreover, the cities with the higher TBC are mostly provincial capitals or regional centers, showing that they act as transfer hubs. The cities with higher WHC are gathered in the eastern and central regions of China, which shows that accessibility for eastern and central cities is significantly better than western and northeast cities.

Secondly, by comparing the centrality results of HSR and air transportation networks, we found that a spatial differentiation characteristic exists, which may be due to the level of economic development of the region. There is little difference between HSR and air transportation in eastern cities because many cities have both higher air and higher HSR rankings. Some cities in the central region have complementary roles in HSR and air transportation networks. However, air transportation is still dominant in western cities, except for some large cities like Chengdu, Chongqing, and Xi'an.

Third, network robustness analysis results showed that the overall efficiency of the multimodal network decreased slightly when some HSR stations or airports with the highest centrality became paralyzed, whether compared to HSR or air transportation networks. That indicated that the multimodal network can maintain network connectivity despite emergencies.

These results revealed that weighted centrality measures are effective in measuring the topological importance of nodes in a weighted network. Besides, some nodes with higher weighted centrality have a more significant influence on network connectivity. The government and related companies should pay more attention to the operational management of these HSR stations and airports. Centrality analysis allows us to evaluate regional disparities in transport network connectivity and accessibility (e.g., cities in western China) and provides useful information on which regions to prioritize for future transport infrastructure development. Besides, since an epidemic can spread quickly via public transportation, such as airplanes and highspeed trains, the government can control passenger flow or block critical HSR stations and airports to slow down or stop an epidemic like COVID-19.

This study has some limitations that should be addressed in future studies. Firstly, real passenger traffic data from HSR in China is not easy to collect. Seating capacity was used instead of estimated passenger traffic as the link weight in the weighted HSR network. Secondly, we investigated the characteristics of static HSR and air transportation networks instead of exploring 
structural changes over the years. According to the Medium-and Long-Term Railway Plan, which calls for an HSR network of $30,000 \mathrm{~km}$ by $2020,38,000 \mathrm{~km}$ by 2025 , and $45,000 \mathrm{~km}$ by 2030 , the expansion of the HSR network in China will accelerate over the next five to ten years. With the expansion of the high-speed network, more HSR lines will be constructed, and more cities will be included in multimodal networks, so the dynamic evolution of networks should be considered in future research by using time series data.

Thirdly, SNA illustrates the network flow and structure, but it does not explain further. Future studies can compare centrality with the comprehensive competitiveness of a city by combining the SNA method and the regression model or gravity model by using population or economic indicators. That can better reflect the impact on city development of a change in the HSR network structure. Besides, this study considered HSR trains, including the C-train, D-train, and G-train, but the different service speeds in these three types of HSR trains may influence passenger choices. Maybe a division into different trains can be used for different weights of networks.

We have tried to investigate the network performance when air and HSR services are interrupted by emergency events, such as extreme weather, natural disasters, or an epidemic. But in the real world, the highway is more likely to be affected (Zhang and Alipour 2020). Therefore, the highway should be considered in the network robustness analysis in future studies. Besides, the duration of disruptive events also impacts passenger travel.

\section{Acknowledgments}

This work was supported by Global Research Network program through the Ministry of Education of the Republic of Korea and the National Research Foundation of Korea (NRF-2017S1A2A2041812).

\section{References}

Albalate, D., Bel, G., Fageda, X., 2015. Competition and cooperation between high-speed rail and air transportation services in Europe. Journal of Transport Geography 42, 166-174.

Albert, R., Jeong, H., Barabási, A. L., 2000. Error and attack tolerance of complex networks. Nature 406, 378-382.

Bagler, G., 2008. Analysis of the airport network of India as a complex weighted network. Physica A: Statistical Mechanics and Its Applications 387, 2972-2980.

Barabási, A. L., Albert, R., 1999. Emergence of scaling in random networks. Science 286, 509-512.

Barrat, A., Barthélemy, M., Pastor-Satorras, R., Vespignani, A., 2004. The architecture of complex weighted networks. Proceedings of the National Academy of Sciences 101, 3747-3752.

Behrens, C., Pels, E., 2012. Intermodal competition in the London-Paris passenger market: High-speed rail and air transport. Journal of Urban Economics 71, 278-288.

Cadarso, L., Marín, Á., Maróti, G., 2013. Recovery of disruptions in rapid transit networks. Transportation Research Part E: Logistics and Transportation Review 53, 15-33.

Cao, W., Feng, X., Jia, J., Zhang, H., 2019. Characterizing the structure of the railway network in China: A complex weighted network approach. Journal of Advanced Transportation 2019, 3928260.

Chen, C., D’Alfonso, T., Guo, H., Jiang, C., 2018. Graph theoretical analysis of the Chinese high-speed rail network over time. Research in Transportation Economics 72, 3-14.

Chen, C. L., 2012. Reshaping Chinese space-economy through high-speed trains: Opportunities and challenges. Journal of Transport Geography 22, 312-316.

Chen, Z., Rose, A., 2018. Economic resilience to transportation failure: A computable general equilibrium analysis. Transportation 45, 1009-1027.

Chi, L. P., Cai, X., 2004. Structural changes caused by error and attack tolerance in US airport network. International Journal of Modern Physics B 18, 2394-2400.

Chung, H. M., Kwon, O. K., Han, O. S., Kim, H. J., 2020. Evolving network characteristics of the Asian international aviation market: A weighted network approach. Transport Policy 99, 299-313.

Clewlow, R. R. L., Sussman, J. M., Balakrishnan, H., 2012. Interaction of high-speed rail and aviation: exploring air-rail connectivity. Transportation research record 2266, 1-10.

De Nooy, W., Mrvar, A., Batagelj, V., 2018. Exploratory Social Network Analysis with Pajek: Revised and Expanded Edition for Updated Software. Cambridge University Press, Cambridge, UK. 
Diestel, R., 1997. Graph Theory: Graduate Texts in Mathematics. Springer, Berlin, Germany.

Du, W. B., Liang, B. Y., Hong, C., Lordan, O., 2017. Analysis of the Chinese provincial air transportation network. Physica A: Statistical Mechanics and Its Applications 465, 579-586.

Du, W. B., Zhou, X. L., Lordan, O., Wang, Z., Zhao, C., Zhu, Y. B., 2016. Analysis of the Chinese Airline Network as multilayer networks. Transportation Research Part E: Logistics and Transportation Review 89, 108-116.

Estrada, E., Rodríguez-Velázquez, J. A., 2005. Subgraph centrality in complex networks. Physical Review E 71, 056103.

Feng, C., Zhu, Q., Yu, B., Zhang, Y., 2017. Complexity and vulnerability of high-speed rail network in China. 2017 36th Chinese Control Conference (CCC), Dalian, China.

Freeman, L. C., 1978. Centrality in social networks conceptual clarification. Social Networks 1, 215-239.

Gao, C., Wei, D., Hu, Y., Mahadevan, S., Deng, Y., 2013. A modified evidential methodology of identifying influential nodes in weighted networks. Physica A: Statistical Mechanics and Its Applications, 392, 5490-5500.

Givoni, M., Banister, D., 2006. Airline and railway integration. Transport Policy 13, 386-397.

Gu, S., Li, K., 2019. Reliability analysis of high-speed railway network. Proceedings of the Institution of Mechanical Engineers, Part O: Journal of Risk and Reliability 233, 1060-1073.

Guimerà, R., Mossa, S., Turtschi, A., Amaral, L. A. N., 2005. The worldwide air transportation network: Anomalous centrality, community structure, and cities' global roles. Proceedings of the National Academy of Sciences 102, 7794-7799.

ICAO [International Civil Aviation Organisation] 2018. The world of air transport in 2017. Available at: https://www.icao. int/annual-report-2017/Pages/the-world-of-air-transport-in-2017-statistical-results.aspx

Jiang, C., Zhang, A., 2014. Effects of high-speed rail and airline cooperation under hub airport capacity constraint. Transportation Research Part B: Methodological 60, 33-49.

Jiang, Y., Yao, B., Wang, L., Feng, T., Kong, L., 2017. Evolution trends of the network structure of Spring Airlines in China: A temporal and spatial analysis. Journal of Air Transport Management 60, 18-30.

Jiao, J., Wang, J., Jin, F., 2017. Impacts of high-speed rail lines on the city network in China. Journal of Transport Geography 60, 257-266.

Kwon, O. K., Lee, S., Chung, H. M., Chhetri, P., Han, O. S., 2019. Network robustness of major Asian Airlines and the impact of airports' brokerage roles. Journal of International Logistics and Trade 17, 89-102.

Latora, V., Marchiori, M., 2001. Efficient behavior of small-world networks. Physical Review Letters 87, 198701.

Lawrence, M. B., Bullock, R. G., Liu, Z., 2019. China's High-Speed Rail Development. World Bank Group, Washington, DC.

Lee, J. Y., 2006. Centrality measures for bibliometric network analysis. Journal of the Korean Society for Library and Information Science 40, 191-214.

Lee, J., 2013. A comparison study on the weighted network centrality measures of tnet and WNET. Journal of the Korean Society for Information Management 30, 241-264.

Li, T., Rong, L., 2020. A comprehensive method for the robustness assessment of high-speed rail network with operation data: A case in China. Transportation Research Part A: Policy and Practice 132, 666-681.

Liu, H., Tian, Z., Huang, A., Yang, Z., 2018. Analysis of vulnerabilities in maritime supply chains. Reliability Engineering \& System Safety 169, 475-484.

Liu, S., Wan, Y., Ha, H. K., Yoshida, Y., Zhang, A., 2018. Impact of high-speed rail network development on airports: Evidence from China and Japan. Social Science Research Network 127: 115-135.

Liu, S., Wan, Y., Zhang, A., 2020. Does China's high-speed rail development lead to regional disparities? A network perspective. Transportation Research Part A: Policy and Practice 138, 299-321.

Lordan, O., Sallan, J. M., Escorihuela, N., Gonzalez-Prieto, D., 2016. Robustness of airline route networks. Physica A: Statistical Mechanics and its Applications 445, 18-26.

Lordan, O., Sallan, J. M., Simo, P., 2014. Study of the topology and robustness of airline route networks from the complex network approach: A survey and research agenda. Journal of Transport Geography 37, 112-120.

Lordan, O., Sallan, J. M., Simo, P., Gonzalez-Prieto, D., 2014. Robustness of the air transport network. Transportation Research Part E: Logistics and Transportation Review 68, 155-163.

Marchiori, M., Latora, V., 2000. Harmony in the small-world. Physica A: Statistical Mechanics and Its Applications 285, 539546.

MLIT [Ministry of Land, Infrastructure, Transport and Tourism], 2012. 新幹線旅客輸送量の推移. Available at: https:// www.mlit.go.jp/en/ 
MOT [Ministry of Transport of the People's Republic of China], 2020. 2019 Statistical Bulletin of China Railway Corporation. MOT, Beijing, China.

Nicosia, V., Criado, R., Romance, M., Russo, G., Latora, V., 2012. Controlling centrality in complex networks. Scientific Reports 2, 218.

Opsahl, T., Agneessens, F., Skvoretz, J., 2010. Node centrality in weighted networks: Generalizing degree and shortest paths. Social Networks 32, 245-251.

Pan, X., Ning, L., Shi, L., 2019. Visualisation and determinations of hub locations: Evidence from China's interregional trade network. Research in Transportation Economics 75, 36-44.

Scott, J., 1988. Social network analysis. Sociology 22, 109-127.

Shen, J., Duan, H., Zhang, B., Wang, J., Ji, J. S., Wang, J., Pan, L., Wang, X., Zhao, K., Ying, B., Tang, S., Zhang, J., Liang, C., Sun, H., Lv, Y., Li, Y., Li, T., Li, L., Liu, H., Zhang, L., Wang, L., Shi, X., 2020. Prevention and control of COVID-19 in public transportation: Experience from China. Environmental Pollution, 266, 115291.

Sheng, W., Teng, S. N., Hui-Jia, L., Xu, J., Ma, H., Xia-li, L., Yang, X., Shen, D., Liu, M., Huang, Z. Y. X., Xu, C., 2019. Hierarchical structure in the world's largest high-speed rail network. PLOS ONE 14, e0211052.

Sun, X., Wandelt, S., Linke, F., 2015. Temporal evolution analysis of the European air transportation system: air navigation route network and airport network. Transportmetrica B: Transport Dynamics 3, 153-168.

Wang, K., Xia, W., Zhang, A., Zhang, Q., 2018. Effects of train speed on airline demand and price: Theory and empirical evidence from a natural experiment. Transportation Research Part B: Methodological 114, 99-130.

Wang, Y., Cullinane, K., 2016. Determinants of port centrality in maritime container transportation. Transportation Research Part E: Logistics and Transportation Review 95, 326-340.

Wilkinson, S. M., Dunn, S., Ma, S., 2012. The vulnerability of the European air traffic network to spatial hazards. Natural Hazards 60,1027-1036.

Xu, Z., Harriss, R., 2008. Exploring the structure of the U.S. intercity passenger air transportation network: A weighted complex network approach. GeoJournal 73, 87-102.

Xu, W., Zhou, J., Qiu, G., 2018. China's high-speed rail network construction and planning over time: A network analysis. Journal of Transport Geography 70, 40-54.

Yu, N., de Jong, M., Storm, S., Mi, J., 2013. Spatial spillover effects of transport infrastructure: Evidence from Chinese regions. Journal of Transport Geography 28, 56-66.

Zhang, J., Hu, F., Wang, S., Dai, Y., Wang, Y., 2016. Structural vulnerability and intervention of high speed railway networks. Physica A: Statistical Mechanics and its Applications 462, 743-751.

Zhang, N., Alipour, A., 2020. Multi-scale robustness model for highway networks under flood events. Transportation Research Part D: Transport and Environment 83, 102281.

Zhang, P., Zhao, Y., Zhu, X., Cai, Z., Xu, J., Shi, S., 2020. Spatial structure of urban agglomeration under the impact of highspeed railway construction: Based on the social network analysis. Sustainable Cities and Society 62, 102404.

Zhou, L., Chen, Z., 2020. Measuring the performance of airport resilience to severe weather events. Transportation Research Part D: Transport and Environment 83, 102362.

Zhou, Y., Sheu, J. B., Wang, J., 2017. Robustness assessment of urban road network with consideration of multiple hazard events. Risk Analysis 37, 1477-1494.

Zhou, Y., Wang, J., Huang, G. Q., 2019. Efficiency and robustness of weighted air transport networks. Transportation Research Part E: Logistics and Transportation Review 122, 14-26. 